\title{
Community management et métiers émergents du numérique
}

Une analyse des représentations par l'étude des référentiels du marketing et de la communication

Valérie Larroche, Enseignant-chercheur en sciences de l'information et de la communication, IUT Lyon3, Elico, valerie.larroche@univ-lyon3.fr 


\title{
Résumé
}

Cet article approfondit les représentations des métiers émergents du numérique par les employeurs annonceurs et les recruteurs d'agence de recrutement. En comparant 12 fiches métiers de différents référentiels en communication marketing publiés entre 2009 et 2015, on constate des stratégies de visibilité qui minimisent les relations permettant la reconnaissance mutuelle d'individus singuliers. Un focus sur le métier de community manager permet d'une part d'observer l'évolution des représentations de ce métier par les recruteurs, et d'autre part d'analyser les relations établies entre le community management et les métiers émergents étudiés. Les référentiels dans cet article sont le matériel de base des analyses. Ils sont considérés comme des dispositifs de confiance (Karpik, 2007) et des outils de traduction (Callon, 1986).

Mots-clés: référentiels métiers, métiers émergents du numérique, dispositif de confiance, community manager, visibilité

\begin{abstract}
This article examines in depth employers' and recruitment agencies' representations of emerging digital professions. Comparing twelve different emerging job's descriptions released in the 2009-2015 period, we put in evidence that visibility strategies are privileged over the mutual recognition between individuals. A focus on community manager allows us firstly, to observe how representations of recruiters are evolving and secondly, to explore relations mainly between community management and other emerging professions. Reference guides in this article are considered as confidence devices (Karpik, 2007) and non-human actors (Callon, 1986).
\end{abstract}

Keywords: skills checklist, emerging professions in numeric fields, confidence devices, community manager, visibility 
Dans le secteur du numérique, on constate la présence de métiers en transformation et de nouveaux métiers qui répondent aux besoins d'adaptation des entreprises à un environnement changeant et à l'intégration des nouvelles technologies de l'information et de la communication. Parmi celles-ci, les médias socionumériques sont des dispositifs technologiques qui sont venus bouleverser les échanges marchands en y introduisant de l'interaction entre les représentants de l'annonceur et les internautes tout en mesurant l'efficacité des actions. L'espace numérique est aujourd'hui à la fois un espace conversationnel où la communication est interactive, notamment sur les médias socionumériques (Stenger, Coutant, 2013) et un espace investi par les préoccupations de mesure marketing. Pour Thomas Stenger et Alexandre Coutant, ces espaces sont investis notamment par les community managers (Stenger, Coutant, 2011). Parallèlement, on peut observer une dynamique des métiers dans le secteur du numérique, révélée notamment par les publications de référentiels, conçus selon une logique de professionnalisation qui fait aussi l'objet de «positionnement concurrentiel, tant sur le « marché » de la formation que sur celui (ou ceux) des professionnels dont les associations et syndicats sont nombreux à se disputer la représentation, voire le contrôle. » (de la Broise, 2013, p. 44).

Dans ce contexte, nous nous sommes demandée comment les référentiels se représentent le community manager au sein de ces différents métiers et quelles évolutions sont observables au cours de la période 2009-2015.

Nous supposons que le community management, métier relationnel, est un métier pouvant être revendiqué par le secteur de la communication. Nous supposons aussi que ce métier a évolué en six ans et que d'autres métiers de communication spécialisés dans le numérique sont apparus.

Dans cet article, nous désignons par le terme de métier un ensemble d'activités, de compétences et de tâches réunies sous une dénomination et intégrée à une fiche métiers rédigée par un organisme reconnu dans le secteur du recrutement (comme l'Apec) ou par les acteurs reconnus par les spécialistes de la communication et du marketing. La notion de métier est donc réduite à une dénomination et ne comporte pas d'éléments relevant de l'identité collective (Osty, 2003). Le métier est perçu au travers d'une fiche métiers, rédigée par des acteurs dotés d'une légitimité sociale (Bouquet, 2014). Les métiers émergents sont réduits ici à ceux désignés comme tels par les référentiels de 2015.

La première partie s'attachera à présenter et à justifier le choix des référentiels sur lesquels porte cette étude et à préciser les fiches métiers que nous avons confrontées. La deuxième partie propose un classement des fiches métiers en fonction des secteurs, précise la présence ou non de compétences info-communicationnelles et discute les représentations des métiers du numérique par les auteurs du référentiel de la communication. Enfin la dernière partie analyse le métier de community manager 
dans l'ensemble des référentiels et étudie l'évolution de ce métier de 2009 à 2015, permettant ainsi d'établir des filiations avec des métiers plus récents.

\section{Méthodologie : corpus et objet d'analyse}

\subsection{Les référentiels}

Les référentiels métiers sont rédigés par des collectifs professionnels influents et comportent des représentations concernant les compétences et le champs des activités numériques. Ces référentiels sont à la fois des « références partagées au sein d'un corps de métiers» (Lépine, Peyrelong, 2012, p.67) et des guides «permettant au salarié de naviguer dans un ensemble d'emplois identifiés » (Osty, 2003, p. 174).

Ces référentiels ont un rôle à jouer dans la représentation professionnelle des activités numériques puisqu'ils sont rédigés de manière concertée par des organismes que nous qualifions de dispositifs de confiance (Karpik, 2007) et de porte-parole (de la Broise, 2013). Ils sont une approche par les compétences qui "permet d'expliciter des contenus professionnels de façon à ce qu'ils soient repérables et lisibles par l'ensemble des acteurs, mais aussi, si possible, au-delà du petit cercle du champ du social» (Kittel, 2014, p. 44). Pour les métiers émergents, il est important d'apparaitre dans des référentiels car c'est une première forme de reconnaissance puisqu'ils ont été identifiés par des acteurs influents.

Nous intéressant tout particulièrement aux activités info-communicationnelles, nous avons retenu comme dispositifs de confiance les organismes fédérateurs et légitimes en France dans ce secteur que sont l'AFCI (Association française de communication interne), l'AACC (l'Association des agences-conseil en communication), le SYNAP (Syndicat national des attachés de presse et des conseillers en relations publics), l'Union des annonceurs et l'UDECAM (Union des entreprises de conseil \& achat média).

Ces organismes se sont fédérés pour publier en novembre 2013 le référentiel des métiers de la communication. Il comporte quarante fiches qui visent à

couvrir l'ensemble des métiers de la communication, afin d'être utiles à tous les professionnels et futurs professionnels de la communication, mais également aux chefs d'entreprise, aux recruteurs (cabinets, DRH), aux pouvoirs publics et organismes officiels (INSEE, Pôle Emploi, ministères, CIO, ONISEP, etc.), ainsi qu'au monde de l'enseignement et de la formation. (Source : 
130 Le communicateur bousculé par le numérique

http://www.uda.fr/sinformer-actualites/actualites/les-metiers-de-lacommunication/ $/)^{82}$

Les syndicats et associations cités ci-dessus sont légitimés par une identité forte, ils sont représentés par des individus reconnus pour leur expertise communicationnelle et informationnelle qui n'est pas nécessairement numérique. A ce sujet, parmi les quarante fiches proposées, seules cinq seront prises en compte dans notre étude : animateur (trice) de communautés, consultant en e-reputation, responsable communication on-line, traffic manager, webmaster. Nous n'avons en effet retenu dans cet article que les métiers qui, dans leur dénomination, relevaient du numérique. Nous avons retenu animateur de communauté car d'autres appellations synonymes étaient mentionnées dans la fiche dont celle de community manager. Certains métiers à responsabilité ne font pas mention du numérique dans leur dénomination mais comportent malgré tout un volet numérique dans les tâches. Nous n'en avons pas tenu compte car ces propos restent très généraux ${ }^{83}$. Enfin, nous avons exclu le chef de projet (print et web) et le responsable des publications (printweb) car le contenu de la fiche exprime surtout des considérations renvoyant à des missions liées aux documents imprimés, le support web étant uniquement mentionné.

Nous avons également étudié certaines fiches-métiers citées dans la cartographie du portail des métiers de l'Internet et plus particulièrement ceux répertoriés dans la rubrique communication-marketing (voir Figure 12) ${ }^{84}$.

Ce portail a été créé par la délégation aux usages de 1'Internet (DUI) ${ }^{85}$ associée à de nombreux partenaires ${ }^{86}$ pour « favoriser une meilleure connaissance de ces métiers et répondre aux besoins de compétences des acteurs de l'économie numérique ${ }^{87}$. Il

${ }^{82}$ Voir aussi (de la Broise, 2013, p. 44-45)

${ }^{83}$ Exemple : «Le responsable de la communication externe supervise les rédacteurs de contenu, les attachés de presse, les chargés de communication online et community managers et le suivi des supports de communication externe hors médias. » (Référentiel de la communication, 2013)

${ }^{84}$ Nous expliciterons dans la partie suivante la manière dont nous avons sélectionné certaines fiches-métier.

${ }^{85}$ Créée en juillet 2003, lors du Comité interministériel pour la société de l'information (CISI), La DUI est aujourd'hui rattachée au Ministère chargé de l'économie, du redressement productif et du numérique.

${ }^{86}$ Les partenaires sont entre autres : L' ACFCI (Assemblée des chambres françaises de commerce et d'industrie), l'ADBS, association des professionnels de l'information et de la documentation, L'APROGED, l'association des professionnels pour l'économie numérique, le CELSA, grande école rattachée à l'université Paris-Sorbonne (Paris IV), l'ACSEL, l'association de l'économie numérique, Cap Digital, le pôle de compétitivité des contenus et services numériques, le CIGREF, réseau de Grandes entreprises, l'ONISEP (Office national d'information sur les enseignements et les professions), etc. Pour obtenir les noms de l'ensemble des partenaires, consulter la page: http://metiers.internet.gouv.fr/partenaires

${ }^{87}$ Citation extraite de la page : http://metiers.internet.gouv.fr/le-portail 
s'agit également d'un outil d'information et d'orientation de référence sur les métiers du web et les formations qui y préparent.

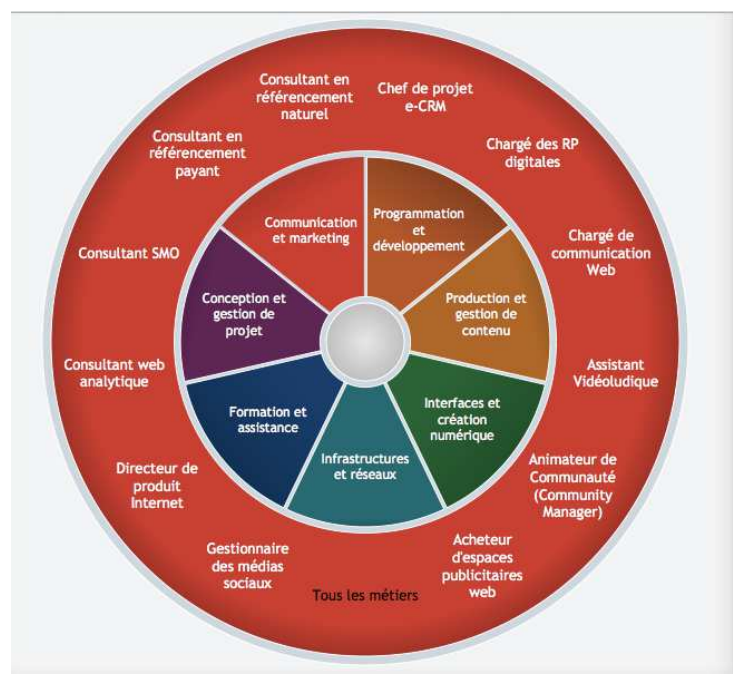

\section{FIGURE 12}

\section{La rubrique communication \& marketing du portail des métiers de l'Internet disponible sur : http://metiers.internet.gouv.fr/}

Certains organismes comme l'ADBS, association regroupant des spécialistes de l'information, proposent une cartographie interactive ${ }^{88}$ qui renvoie aux fiches métiers proposées par le référentiel des métiers de l'Internet. On voit donc que ce portail possède une certaine notoriété.

Les référentiels dont il vient d'être question sont réalisés par des professionnels du secteur de la communication, du digital ou de l'information ${ }^{89}$. Nous ajoutons à ce

\footnotetext{
${ }^{88}$ Proposée comme support de communication lors du salon Documentation des 20 et 21 mars 2013, cette cartographie métiers est « une esquisse d'un redécoupage de la fonction information autour de 7 grandes familles: Gestion des ressources informationnelles, Records Management, Gestion des connaissances, Architecture de l'information, Communication web, Edition numérique, Veille/Etude ». ADBS, Carte métiers ADBS 2013, [en ligne], disponible sur http://www.adbs.fr/carte-metiers-adbs-2013-132079.htm (consulté le $1^{\text {er }}$ décembre 2014)

Consulter la cartographie à l'adresse suivante : http://www.adbs.fr/brochure-presentation-adbs-etcartographie-metier-127345.htm

${ }^{89}$ Nous avions au préalable recherché des cartographies produites dans le secteur de la publicité. Nous en avons trouvé une, publiée par l'Observatoire des métiers de la publicité mais cette cartographie interactive (pour certains métiers) ne répertorie qu'un métier numérique, le consultant In-
} 
corpus le référentiel des métiers de l'Internet publié récemment par l'Observatoire du marché de l'emploi cadre de l'Apec ${ }^{90}$ ). En effet, l'Apec a créé

\begin{abstract}
une série de fiches décrivant dans le détail, les missions, les profils, les compétences de 12 métiers incontournables, illustrés par le témoignage de cadres en activité, précise Sylvie Delattre, responsable des études sur les métiers à l'Apec (Marketing digital : des métiers cadres en constante mutation, 23 juin 2015, disponible sur: https://recruteurs.apec.fr/Recrutement/Observatoire-de-1emploi/Les-etudes-Apec-par-thematique/Metiers-et-competences/Marketingdigital-des-metiers-cadres-en-constante-mutation, consulté le 29 juillet 2015)
\end{abstract}

L'Apec précise que ce référentiel s'adresse à la fois aux jeunes issus de l'enseignement supérieur ou à des cadres plus expérimentés, pour leur permettre d'adapter leurs candidatures aux besoins des entreprises. Il s'adresse aussi aux professionnels des ressources humaines, pour les aider à mieux définir leurs postes pour une démarche de recrutement ou de développement des compétences des collaborateurs. Il apparaît clairement comme un document de communication qui cartographie les dénominations métiers, les compétences et tâches associées

\title{
1.2. Les fiches-métiers
}

Les référentiels employés dans cette étude sont donc des répertoires constitués de fiches-métier, proposés comme des références aux acteurs du recrutement (services $\mathrm{RH}$, employeurs, cabinets de recrutement, candidats, service d'orientation). Ils constituent un inventaire de l'emploi, des activités et des compétences liées à ces activités.

Les référentiels comportent des fiches-métiers qui sont composées de plusieurs rubriques :

- Une rubrique «activité » qui se définit selon la Commission nationale de la certification professionnelle (CNCP) comme l'ensemble logique et/ou chronologique (processus) de tâches effectivement réalisées par une personne et concourant à une ou plusieurs fonctions dans une entreprise, selon des conditions d'exercice identifiées ${ }^{91}$;

- Une rubrique «tâche» qui décrit ce qu'il y a à faire, exprimé sous forme de consigne ;

ternet, qui ne fait référence à aucun contenu, donc inutile pour notre analyse. La cartographie est disponible sur http://www.metiers-publicite.fr/representation_interstructure (consulté le 29 juillet $2015)$.

${ }^{90}$ Cet observatoire a pour mission d'anticiper les évolutions dans un programme annuel d'études et de veille. Il publie des grandes enquêtes annuelles (recrutements, salaires, métiers et mobilité professionnelle des cadres, insertion professionnelle des jeunes diplômés, etc.).

${ }^{91}$ Voir le glossaire du CNCP : http://www.cncp.gouv.fr/glossaire 
- Une rubrique « compétence » dans laquelle les capacités à agir sont listées. Les compétences répertorient les connaissances, les savoir-faire et les comportements mobilisés lors de l'activité réalisée dans un contexte professionnel (le Boterf, 1997).

Chaque fiche possède comme titre la dénomination d'un métier. D'autres désignations sont proposées comme synonyme.

Pour le référentiel de l'Apec, les fiches métiers sont classées en trois catégories (Référentiel Apec, Les métiers du marketing digital, 2015, p. 6) :

- Les métiers de la donnée (data), de la stratégie et de l'innovation (marketing de la stratégie digitale, marketing business analyst, data scientist/data miner)

- Les métiers du marketing opérationnel (marketing relationnel/CRM, marketing opérationnel/digital, traffic manager)

- Les métiers du marketing marque, produit, prix (chef de produit Web, brand manager, community manager, social media marketing manager, content manager, analyst revenue manager)

Pour ce référentiel, les fiches comportent d'autres informations intéressantes comme le rattachement hiérarchique, les relations fonctionnelles et des témoignages de personnes exerçant ce métier.

Aux référentiels présentés dans la partie précédente, nous avons ajouté celui d'un cabinet de recrutement spécialisé dans le marketing, la communication et le digital : Elaee. Il s'agit d'une agence de recrutement dédiée à «toutes les fonctions en agence ou en service de com' ou marketing intégrés ${ }^{92}$. Les consultants web, marketing digital (MD), Customer Relationschip Management (CRM) - en français, Gestion de la Relation client, Search Engine Optimisation (SEO) - en français référencement naturel, Social Media Optimization (SMO) - en français Optimisation pour les médias sociaux ou référencement social - font partie des profils les plus recrutés d'après l'agence. Comme notre but était de caractériser les compétences info-communicationnelles, il nous a semblé intéressant de nous référer également à une agence de recrutement spécialisée en communication. On peut en effet espérer trouver dans leur référentiel des caractéristiques précises concernant la communication.

Elaee édite un webzine dénommé «magazine de l'emploi dans la création, la communication, le marketing et le digital» dans lequel sont diffusées des fichesmétier $^{93}$ originales : elles sont rédigées sous forme d'articles par les experts du

\footnotetext{
92 «Les métiers qu'Elaee recrute », disponible sur : http://www.elaee.com/cabinet-de-recrutementcommunication

${ }_{93}$ Elaee élabore régulièrement des fiches métiers sur la page http://www.elaee.com/fiches-metiers. Les fiches répertoriées à la date du 15 juin 2015 sont : Chargé de trade marketing, Directeur artistique (DA), Responsable e-commerce, Chargé de communication, Data scientist, Chef de projet digital, Planneur stratégique, Ergonome web, E-reputation manager
} 
recrutement de l'agence Elaee au gré des envies des responsables du magazine. Les articles ne sont pas systématiquement datés. En plus des missions, des aptitudes et compétences, l'auteur (un des experts de la société Elaee) ajoute à chaque fiche une rubrique concernant la place du métier dans l'organisation, le salaire, les formations et son avis sur le métier. Certaines de ces fiches sont reprises par le site « Jobmarketingvente.com»: Chargé de trade marketing, Directeur artistique (DA), Responsable e-commerce, Chargé de communication, Data scientist, Chef de projet digital, Planneur stratégique, E-reputation manager, Directeur conseil. Celles présentes uniquement sur le site Elaee sont : Attaché de presse, chef de produit, chef de projet, chef de projet web, chef de projet web éditorial, community manager, concepteur-rédacteur, directeur clientèle, directeur de communication, directeur marketing, e-reputation manager, ergonome web, journaliste, motion designer, styliste, traffic manager, webmarketer, webmaster, planneur stratégique.

Nous avons considéré ce cabinet de recrutement comme un dispositif de confiance (Larroche, 2014), dont la légitimité provient de son expertise en matière de recrutement combinée à des expertises numériques.

\subsection{La sélection des fiches-métiers étudiées}

Notre objectif était d'établir un comparatif des fiches-métiers du référentiel de la communication, du portail des métiers de l'Internet, du référentiel de l'Apec et des articles fiches-métier proposés par Elaee en ne retenant que les métiers liés au digital. Pour établir cette liste, nous nous sommes appuyée à la fois sur le référentiel publié par l'Apec et sur une étude publiée en février 2015 par AddedValue car elle a été commanditée par l'IAB ${ }^{94}$ (Interactive Advertising Bureau), association créée en 1998 pour structurer le marché de la communication sur Internet, favoriser son usage et optimiser son efficacité. Cette étude ${ }^{95}$ a en effet pour ambition de dresser la

94 Interactive Advertising Bureau France (IAB France), Etude sur les métiers et les comptétences, de la transition numérique dans le secteur du marketing et de la communication, disponible sur : http://www.iabfrance.com/presse/communiques-de-presse/les-metiers-du-marketing-et-de-lacommunication

${ }^{95}$ Cette étude a été réalisée à l'aide d'un comité scientifique réuni autour de Jean-Paul Aimetti, professeur titulaire de la chaire études et stratégie marketing du CNAM, regroupant des représentants de chaque partenaire de l'étude : L'ACSEL - l'association de l'économie numérique qui réunit les acteurs engagés dans la transformation digitale, le SNCD, syndicat de la communication directe, le CPA - Collectif de la performance et de l'acquisition - l'Efap, école de communication, Aquent (agence de talents spécialisée depuis trente ans sur les métiers du marketing, de la création et du digital), le Groupe La Poste et Microsoft. Une étude documentaire a été effectuée puis une étude qualitative a été réalisée à partir de l'analyse d'entretiens menés auprès de parties prenantes (agences de publicité, annonceurs, centrales d'achat d'espaces, régies, éditeurs, sociétés de conseil, prestataires, etc.) et de l'analyse des groupes de discussion regroupant des acteurs des différents métiers. Enfin une étude quantitative administrée en ligne auprès des acteurs de l'industrie (400 répondants) 
feuille de route des métiers et compétences du marketing et de la communication de demain. Nous reproduisons ci-après un des résultats de l'étude, concernant les dix métiers amenés à perdurer dans les années à venir (voir Figure 13).

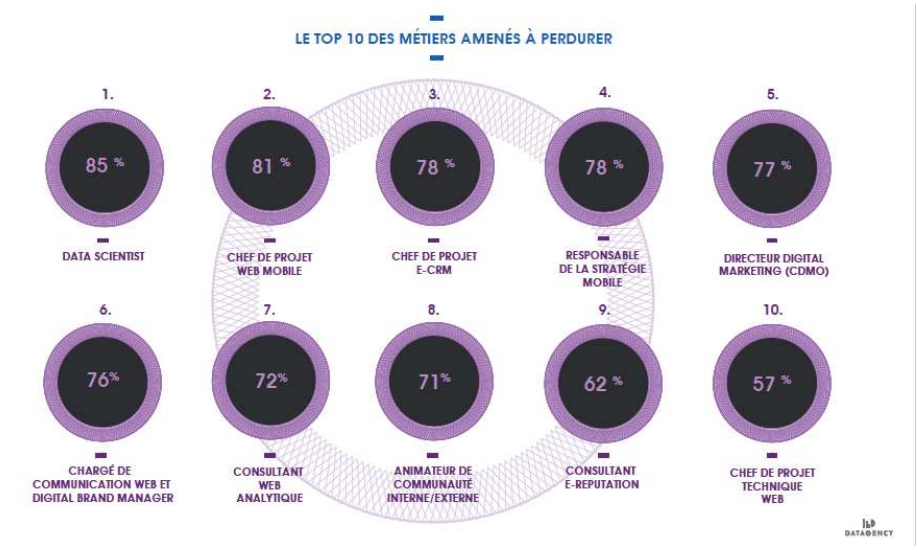

\section{FIGURE 13}

les métiers du digital amenés à perdurer cités par l'étude IAB (p. 22)

En plus de ces dix métiers, nous avons ajouté trois métiers cités par le référentiel des métiers de l'Apec paru en 2015, à savoir le traffic manager, l'analyst revenue et le chef de projet web. En figure 3 ci-après, dans la première colonne, est listé l'ensemble des métiers cités par l'Apec et l'IAB sur lequel a porté notre réflexion.

\section{Les résultats de la confrontation des fiches métiers}

\subsection{Le classement des fiches métiers}

Nous avons souhaité observer en priorité les compétences infocommunicationnelles pour cet article. Les métiers présents dans la figure 2 ont fait l'objet d'un classement présenté en figure 3. Nous avons cherché la présence de compétences communicationnelles dans les métiers sélectionnés. Ceux barrés dans

a été réalisée. En savoir plus sur http://www.iabfrance.com/presse/communiques-de-presse/lesmetiers-du-marketing-et-de-la-communication\#2wv2PxuwJ9fyZDJQ.99 
la figure 3 sont ceux pour lesquels nous n'avons pas approfondi les compétences communicationnelles, les considérant absentes ou marginales.

Notre premier critère de classement a permis de rassembler les fiches métiers en lien avec les compétences info-communicationnelles détenues par des experts en communication, d'après Lucia Granget ${ }^{96}$. Nous les avons regroupées sous l'intitulé «métiers de la communication ». Pour Lucia Granget, les encadrants des services de communication dans les universités associent une communication conversationnelle avec la mission de transmission de la voix officielle. Elle met en avant deux fonctions portées par les communicateurs, celle d'infostratège qui allie surveillance de l'e-réputation et repérage des leaders et des relais d'opinion et celle de community manager qui gère la présence de l'université sur les réseaux sociaux. Nous avons donc créé deux sous-catégories dans notre classement présenté en figure 3. La première regroupe les métiers liés à la fonction d'infostratège. Le chargé de communication web et digital brand manager a été associé au responsable de la communication on line, le consultant e-reputation, a été associé au social media marketing manager. Nous les avons regroupés car le référentiel de l'Apec les a associés dans un schéma où il confronte les métiers de 2015 aux métiers cités dans des référentiels antérieurs (2013) (Apec, 2015, p. 22). L'Apec considère notamment le social media manager comme une évolution du métier de consultant en ereputation. Ces métiers comprennent une dimension stratégique pour rendre visible la société ou la marque sur les canaux numériques. Cela relève d'une visibilité médiatisée, celle qui par «l'usage des médias de communication contribue à l'attention publique et engage l'intervention de tiers sous forme de supports symboliques, de techniques, d'images ou de sons » (Voirol, 2005, p.110). D'autres référentiels mentionnent clairement cette dimension de relation publique, notamment le portail des métiers de l'Internet avec le métier de responsable des relations publiques digitales que nous avons associé au métier de chargé de communication web. Nous y reviendrons en dernière partie.

L'autre sous-catégorie regroupe les métiers liés à la présence sur les médias socionumériques et dont la vocation est essentiellement conversationnelle ou éditoriale. Il s'agit de l'animateur de communautés et du content manager. L'un a pour mission essentielle d'inciter aux échanges, l'autre d'écrire ou de faire écrire de manière à susciter des commentaires ou le relais des billets.

Nous avons créé une deuxième catégorie qui réunit les métiers du marketing. Pour cela, nous avons considéré les métiers liés au traitement des données et ceux liés à des mesure de rentabilité comme relevant de cette catégorie. Nous avons intégré les

\footnotetext{
${ }^{96}$ Nous avons choisi de nous appuyer sur le texte de Lucia Granget pour caractériser les compétences info-communicationnelles car elle a effectué des entretiens compréhensifs auprès d'encadrants des services communication des universités françaises en 2002 et 2004 (vingt interviewés), en 2008 (quinze interviewés) et 2010 (douze interviewés), ce qui lui donne une vision évolutive des compétences digitales.
} 
métiers de consultant web analytics, d'analyst revenue et de data scientist dans cette catégorie. Données et statistiques sont les ingrédients de ces métiers, qui ne correspondent donc pas à des préoccupations communicationnelles, au sens interactionnel. Nous n'y reviendrons pas dans la suite du présent article. C'est pourquoi ils apparaissent barrés dans la figure 3 .

Nous avons classé deux métiers marketing à part car l'un est cité dans le référentiel de la communication et dans une fiche du webzine Elaee. Il s'agit du traffic manager. L'autre comporte une dimension relationnelle. Il s'agit du chef de projet e-crm (Customer Relationship Management ou gestion de la relation clients).

Il gère la relation client sur Internet. En étroite relation avec les équipes informatique, marketing et vente, il assure le marketing relationnel pour l'ensemble des plates-formes numériques d'une entreprise et met en oeuvre des campagnes de gestion de la relation client destinées au Web ( portail des métiers de l'internet, chef de projet e-crm, < $2012^{97}$ ).

Nous avons intitulé une troisième catégorie «métier info-technique de l'Internet », qui contient le chef de projet technique web, anciennement webmaster. Les compétences du webmaster sont aujourd'hui réparties dans d'autres métiers cités dans le tableau. Le référentiel de la communication comporte une fiche sur ce métier qui nous a permis de justifier l'exclusion de ce métier des préoccupations communicationnelles :

En fonction de l'environnement dans lequel il travaille, ses responsabilités peuvent être d'ordre technique à travers la maîtrise d'outils de publication web, de gestion de contenus, des langages spécifiques (html...), de bases de données, de suivi de statistiques et référencements, mais aussi parfois d'ordre éditorial, attachées à l'actualisation comme à la production de contenus simples ». (Référentiel de la communication, 2013, webmaster)

Pour les auteurs du référentiel de la communication, le webmaster est polyvalent et possède des compétences attribuées dans notre tableau au responsable de contenus numériques (rédaction de contenus), au traffic manager (statistique de connexion, référencement), au community manager (rôle de contribution, de validation et d'animation), ce que précise la fiche du référentiel de la communication.

L'augmentation de la taille des sites web comme la diversité des objectifs qu'ils visent et des fonctionnalités qu'ils proposent contribue à segmenter de façon progressive la fonction de webmaster au profit de profils plus spécialisés dont le domaine couvre de façon respective et distincte la dimension éditoriale, la

\footnotetext{
${ }^{97}$ Il n'y a pas de mise à jour de la fiche. Nous avons indiqué la date du commentaire le plus ancien.
} 
138 Le communicateur bousculé par le numérique

dimension d'animation, la dimension technique, etc.» (Référentiel de la communication, 2013, webmaster)

La dimension technique englobe en fait la notion de chef de projet technique web. Nous ne traiterons pas ce métier dans la suite car il ne contient finalement que la dimension technique du site.

Enfin la dernière catégorie comporte une dimension stratégique excluant les dimensions de présence et de visibilité intégrées dans les métiers de la communication par Lucia Granget. Le directeur marketing digital est le plus englobant.

Le responsable de la stratégie digitale a pour mission de développer et déployer la stratégie multicanal de l'entreprise. Il conçoit avec ses équipes internes/externes de nouveaux produits et services digitaux et vise ainsi à maximiser les points de contacts entre l'internaute et la marque (Apec, 2015, p. 23).

La dimension stratégique de ce métier dépasse le secteur de la communication. Ce responsable est en relation hiérarchique ou fonctionnelle avec des stratèges spécialisés en communication web ou en médias socionumériques. Nous n'approfondirons pas les compétences communicationnelles de ce métier considérant que l'aspect stratégique dévolu à la communication est contenu dans le métier de chargé de communication web intégré à la première catégorie intitulée «métiers liés à la fonction d'info-stratège ».

Les autres métiers classés dans cette catégorie sont le responsable marketing mobile et le chef de projet web mobile. Les deux permettent d'intégrer les technologies mobiles, devenues incontournables pour diffuser des produits et services notamment sous forme d'applications mobiles. Ces métiers sont «à l'interface de la communication, de l'informatique, et de la maîtrise d'ouvrage ». (Apec, 2013, p. 12). 


\begin{tabular}{|c|c|}
\hline $\begin{array}{l}\begin{array}{l}\text { Classement des métiers de la } \\
\text { figure } 2\end{array} \\
\end{array}$ & Commentaires \\
\hline \multicolumn{2}{|r|}{ Métiers de la communication (Granget) } \\
\hline \multicolumn{2}{|c|}{ Métiers liés à la fonction d'info stratège (Granget) } \\
\hline $\begin{array}{l}\text { Chargé de communication web et digital| } \\
\text { brand manager, responsable de marque } \\
\text { en ligne, responsable de la } \\
\text { communication on line, responsable du } \\
\text { marketing opérationnel }\end{array}$ & 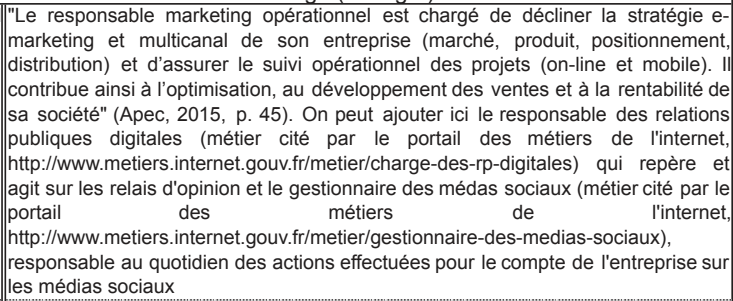 \\
\hline $\begin{array}{l}\text { Consultant e-reputation, social media } \\
\text { marketing manager }\end{array}$ & $\begin{array}{l}\text { Surveillance des propos tenus sur la société, sur ses marques, ses produits et } \\
\text { ses services. Actions de visibilité }\end{array}$ \\
\hline \multicolumn{2}{|c|}{ Métiers liés à la fonction de présence de la société ou de la marque sur les médias sociaux } \\
\hline $\begin{array}{l}\text { Animateur de communauté } \\
\text { interne/externe, community manager } \\
\text { Responsable de contenu numérique, } \\
\text { content manager }\end{array}$ & $\begin{array}{l}\text { Anime les échanges, porte-parole de la société ou de la marque. } \\
\text { Rédaction permettant de susciter les échanges (commentaires, relai du billet...), } \\
\text { garant de la cohérence des contenus. }\end{array}$ \\
\hline \multicolumn{2}{|r|}{ Métiers du marketing } \\
\hline \multicolumn{2}{|r|}{ Métiers du marketing liés à la communication } \\
\hline $\begin{array}{l}\text { Chef de projet e-crm, responsable } \\
\text { marketing e-crm, responsable marketing } \\
\text { relationnel et CRM }\end{array}$ & $\begin{array}{l}\text { Dimension conversationnelle entre le client, le consommateur et l'annonceur. Le } \\
\text { responsable du marketing relationnel met en œuvre un ensemble d'outils destinés } \\
\text { à entretenir des relations individualisées et interactives avec les clients et à } \\
\text { développer la connaissance client: gestion d'une base de données CRM, } \\
\text { organisation des campagnes de recrutement et de fidélisation, suivi des résultats } \\
\text { (Apec, 2015, p. 51). }\end{array}$ \\
\hline Trafic manager, référenceur web & $\begin{array}{l}\text { un métier cité dans le référentiel de la communication. Les compétences liées à ce } \\
\text { métier ne sont pas citées par Granget. Ce métier relève du contrôle et de la } \\
\text { mesure et permet de réorienter les stratégies en fonction des analyses. }\end{array}$ \\
\hline \multicolumn{2}{|r|}{ Métiers de la donnée } \\
\hline Gonsultan analytique & Métier lié à l'analyse de données pour vérifier l'efficacité des actions. \\
\hline Analyst revenue, yield manager & $\begin{array}{l}\text { Optimiser les revenus d'une société, c'est-à-dire, vendre au meilleur prix les } \\
\text { services pour atteindre une rentabilité maximale. Métier essentiellement répandu } \\
\text { dans le secteur du tourisme. }\end{array}$ \\
\hline Datareientist & \begin{tabular}{|l} 
"Le Data scientist est un spécialiste de la science des données. II analyse les \\
datas (concernant les clients, les prospects, les employés, etc.) que l'entreprise \\
récupère par différents canaux et la restitue sous forme de prospective, de \\
conseils, d'améliorations du produit, du service, de la formation en interne, de \\
l'efficacité de l'entreprise, de sa performance" (Extrait fiche métier Elaee, \\
disponible sur http://www.elaee.com/fiches-metiers/fiche-metier-data-scientist). \\
\end{tabular} \\
\hline \multicolumn{2}{|r|}{ Métiers info-techniques de l'internet } \\
\hline 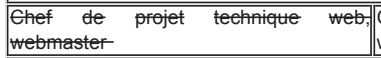 & $\begin{array}{l}\text { Coordination d'équipe technique permettant à l'infrastructure de fonctionner ( site } \\
\text { web, médias sociaux...) }\end{array}$ \\
\hline \multicolumn{2}{|c|}{ Métiers liés à la conception des stratégies digitales (hors fonction d'info-stratège et de stratégie de présence) } \\
\hline Respor de strágie mile & $\begin{array}{l}\text { Le responsable de la stratégie mobile a pour mission d'intégrer les technologies, } \\
\text { applications, développements liés au mobile dans toute l'entreprise : c'est un } \\
\text { métier dont l'interface se situe entre la communication, l'informatique, et la } \\
\text { maîtrise d'ouvrage. Il propose une palette d'applications mobiles en lien avec les } \\
\text { produits et services de l'entreprise, en appui de la stratégie marketing. (Apec, } \\
2013, \text { p.12) }\end{array}$ \\
\hline Eireeteur digital marketing & \begin{tabular}{|l} 
"Le responsable de la stratégie digitale a pour mission de développer et déployer \\
la stratégie multicanal de l'entreprise. II conçoit avec ses équipes \\
internes/externes de nouveaux produits et services digitaux et vise ainsi à \\
maximiser les points de contacts entre l'internaute et la marque" (Apec, 2015, p. \\
23). Dimension stratégique dépassant le secteur de la communication.
\end{tabular} \\
\hline $\begin{array}{l}\text { Ehef de prejet } \\
\text { produit marketing web }\end{array}$ & \begin{tabular}{|l} 
Le chef de projet coordonne des équipes qui peuvent comprendre un spécialiste \\
de la communication. "Le chef de produit web/mobile a pour mission de définir, \\
concevoir et mettre en œuvre de nouveaux services digitaux web et mobile ou \\
d'améliorer l'offre produit existante dans le respect de la stratégie marketing de \\
l'entreprise". (Apec, 2015, p. 67)
\end{tabular} \\
\hline
\end{tabular}

Figure 14

Classement des métiers cités dans l'étude IAB et dans le référentiel de l'Apec (2015) en fonction de critères communicationnels 


\subsection{Les niveaux de responsabilités}

Nous pouvons remarquer que les métiers que nous avons rapprochés dans l'étude IAB et dans le référentiel de l'Apec comportent des expressions du type " responsable», "chef de projet», «chargé de» qui désignent des degrés de responsabilités différents. Le chef de projet est un terme générique qui comporte un rôle de coordination et d'animateur d'équipe mais aussi de conduite de projet avec la prise en compte du suivi technique, de la gestion des problèmes du quotidien, de la répartition du budget, des délais à respecter et des ressources humaines à allouer. Il est le responsable de l'état d'avancement d'un projet. Comme le montre la figure 3, il existe des chefs de projet dans différents domaines d'où les dénominations suivantes : Chef de projet web mobile, Chef de projet E-crm, Chef de projet en marketing relationnel, le terme accolé à chef de projet précisant la nature du projet dont est responsable ce chef de projet. Il est un véritable médiateur, apte à dialoguer avec différents acteurs (des experts en marketing, des communicants, des informaticiens, des veilleurs, des experts de la relation client, des experts du mobile, etc.). Un chef de projet est une fonction transversale que l'on retrouve dans différents domaines.

Nous avons confronté la dénomination «chef de projet» aux termes de « responsable », de « directeur », de « manager », de « chargé de », de « consultant» intégrés à la désignation d'une fiche métier. Les mots « responsable» ou «manager» permettent d'intégrer des fonctions stratégiques au métier. Les expressions « chargé de, gestionnaire ou animateur » insistent sur les fonctions plus opérationnelles, le consultant regroupant les deux niveaux.

Patrice de la Broise avait déjà remarqué lors d'une étude sur « les dircoms » des niveaux de responsabilité variés :

trois niveaux de responsabilité (politique, fonctionnel, opérationnel) qui selon la taille de la structure, conduisent à situer et à qualifier diversement les professionnels de la communication.[...] Le chargé (ou responsable) de communication opère bien plus souvent en qualité de maître d'œuvre, entendu comme celui qui recherche une solution technique permettant la réalisation d'un programme qu'en qualité de maître d'ouvrage (de la Broise, 2006, p. 70) ${ }^{98}$.

Le fait d'accoler le terme « chargé de » ou « responsable» donne une indication sur le degré d'autonomie de l'individu. Le « chargé de » dépend d'un responsable

\footnotetext{
${ }^{98}$ Patrice de la Broise ajoute que « le dircom » est une appellation réservée aux cadres dirigeants opérant dans les grandes structures, souvent issus de grandes écoles (de commerce) et utilisant la fonction communication comme un tremplin vers d'autres directions (Ressources humaines, marketing, etc.). (de la Broise, 2006, p. 70)
} 
qui peut être «directeur» ou «responsable marketing, communication ou informatique ». Dans la figure 3, nous avons pu constater que les degrés de responsabilités concernant les métiers dévolus à la communication numérique ne sont pas encore bien établis. On peut retrouver sur une même ligne la notion de chef de projet et de responsable (voir par exemple la liste démarrant par le chargé de communication, qui comprend aussi le grade de responsable de communication on line).

\subsection{Les représentations des métiers du numérique par référentiel de la communication}

Comme nous nous sommes intéressée aux compétences communicationnelles, il nous a semblé pertinent d'analyser plus finement la vision des métiers de la figure 3 par les communicants. Parmi les métiers cités dans la figure 3, seuls quatre métiers sont détaillés dans le référentiel de la communication, à savoir le community manager, le responsable de la communication on line, consultant en e-réputation et le traffic manager ${ }^{99}$. Les missions du traffic manager (référentiel de la communication, 2013) sont succintes et réduites à la gestion des publicités sur le web alors que le référentiel de l'Apec y ajoute une mission beaucoup plus large d'optimisation de « tous les leviers d'acquisition de trafic sur le web (référencement, partenariats, etc.) afin de donner de la visibilité au site, de générer le maximum de trafic et de chiffre d'affaires" (Apec, 2015, p. 57). Le référentiel de la communication donne donc une idée réductrice du métier de traffic manager.

Quant au métier de responsable de la communication en ligne et au consultant ereputation, ils ont des missions similaires. Nous avons trouvé exactement le même texte de présentation à une phrase près; le responsable on line tout comme le consultant en e-reputation définit la politique de communication on line et a un rôle de chef de projet. Il établit des stratégies d'influence en sélectionnant les relais d'opinion sur lesquels porteront les actions de communication.

Que ce soit en élaborant les stratégies de communication d'une marque sur le web ou en intégrant le web comme outil dans une stratégie globale, il(elle) doit parfaitement connaître les acteurs les plus influents du web ou les lieux d'expression incontournables (leaders d'opinions, blogs, réseaux sociaux)

\footnotetext{
${ }^{99}$ Le référentiel de la communication cite aussi le webmaster, le responsable des publications (print/web) et le chef de projet (print/web). Nous n'avons pas intégré les deux derniers au tableau de la figure 3 car les missions dévolues au responsable des publications ou au chef de projet édition ne sont pas spécifiques aux contenus digitaux. Seul le terme web permet d'intégrer la prise en compte d'écrits numériques. Pour le chef de projet édition il est fait mention du respect des règles d'écriture on line.
} 
142 Le communicateur bousculé par le numérique

(Référentiel de la communication, responsable de la communication on line, et consultant e-reputation, 2013)

Cette citation reprend des éléments de la fiche de responsable des relations publics digitales (portail des métiers de l'Internet, fév . 2012) ${ }^{100}$, qui repère et agit sur les relais d'opinion.

C'est finalement le community manager qui nous a paru le plus en adéquation avec la communication interactionnelle. Mais même si l'échange, la fiabilité des informations sont les maîtres mots de l'animateur de communautés, il reste contraint par des visions marketing, réduisant le dialogue et la gestion des relations à une recherche de visibilité médiatique (Heinich, 2012) servant les stratégies d'influence envisagées par le responsable de la communication en ligne. Il définit certes la cohérence du contenu éditorial, mais est contraint par des critères d'efficacité liés non pas à la qualité rédactionnelle et à la satisfaction des publics, mais à la viralité du message.

il recense, écoute et formalise les besoins éditoriaux de l'entreprise. Il veille au respect des bonnes pratiques validées dans la charte éditoriale. [...] Il suit et exploite les statistiques de publication et d'utilisation des contenus et intervient sur des actions correctives éventuelles (référentiel de la communication 2013).

La citation intègre aux tâches dévolues au community manager des mesures de rentabilité, seuls arguments convaincants pour les dirigeants (Berland, ChevalierKuszla, Sponem, 2008). Les médias socionumériques (Stenger, Coutant, 2013), plateformes sur lesquelles le community manager travaille, permettent des communications interactionnelles entre l'annonceur et ses parties prenantes (notamment les consommateurs) qui établissent des relations mutuellement profitables.

Les annonceurs sont le plus souvent à l'instigation des communautés auxquelles ils participent. C'est le cas des communautés de marque conçues en général par le service marketing des entreprises, dont les objectifs sont d'une part de faire connaître et aimer la marque et d'autre part de fidéliser les consommateurs. Elles sont clairement à finalité marchande et relèvent de la catégorie des communautés d'intérêt (Stenger \& Coutant, 2013). Un annonceur qui crée une communauté de marque n'a pas pour ambition de créer une véritable communauté mais c'est plutôt

une tentative de contrôle ou de reprise en main de l'information circulant sur Internet dans les dispositifs de communications partagés » (Galibert, 2014, p. 118) et une volonté d'intégrer " en amont des clients dans la production des outils et services qu'ils consommeront (Galibert, 2014, p. 118).

${ }^{100}$ Il n'y a pas de date de mise à jour. Le commentaire le plus ancien date de février 2012. 
Le modèle de communication bidirectionnelle symétrique de Grunig et Hunt $(1984)^{101}$ est le modèle théorique de référence des relations publiques développées sur les médias socionumériques et le seul acceptable par les directions. Il se rapproche des caractéristiques données par les promoteurs du marketing de l'engagement sur les réseaux sociaux pour qui ce type de marketing « repose sur le fait que l'entreprise doit ouvrir le dialogue et créer une coopération interactive à double sens avec les consommateurs » (Hossler, Murat, \& Jouanne, 2014, p.165) dans le but de les fidéliser ou de transformer des internautes en consommateur.

Finalement, la vision projetée par le référentiel de la communication est une représentation très marketing de la communication avec des préoccupations de stratégies d'influence et de visibilité. Il est vrai que le référentiel étudié est écrit par des représentants de l'AFCI (Association française de communication interne), de l'AACC (l'Association des agences-conseil en communication), du SYNAP (Syndicat national des attachés de presse et des conseillers en relations publics), de l'Union des annonceurs, et l'UDECAM (Union des entreprises de conseil \& achat média), des organismes défendant des consultants, des annonceurs contraints par des logiques d'efficacité et de retour sur investissement.

\begin{abstract}
Pour les dirigeants, l'idée persiste que la communication est d'abord une fonction marketing de ciblage des publics, qu'elle doit produire des messages, favoriser la circulation de l'information et travailler l'influence et l'écoute (Brulois \& Charpentier, 2013, p. 37).
\end{abstract}

Nous avons également constaté que les communicants n'ont pas investi de manière significative les métiers du numérique. Ils n'en ont pas une connaissance très poussée. Ils intègrent le numérique à leur stratégie traditionnelle de communication sans en définir clairement les spécificités. Le seul métier véritablement connu par les professionnels de la communication intègre des contraintes d'efficacité, illustrant l'acceptation des contraintes marketing au détriment d'un véritable échange conversationnel. L'animateur de communauté a pour objectif de maintenir une image favorable par des procédés contrôlables, très loin finalement de la communication d'interaction réduisant de fait la communication à des techniques de persuasion (Dacheux, 2001, p. 414) et d'influence, bien loin de la communication définie comme « relation humaine qui implique l'existence d'autrui » (Dacheux, 2001, p. 415) et la reconnaissance mutuelle d'individus singuliers.

\footnotetext{
${ }^{101}$ Grunig et Hunt ont développé quatre modèles : agent de presse/promotion, information du public, communication asymétrique bidirectionnelle et communication symétrique bidirectionnelle (Grunig \& Hunt, 1984, p. 21-43). Voir pour une synthèse : (Charest, 2013).
} 


\section{L'évolution du métier de community manager au travers des référentiels}

Nous avons focalisé jusqu'ici notre attention sur le référentiel de la communication paru en 2013 et sur le référentiel de l'Apec paru en 2015. De fait, les référentiels que nous avons choisis ont été publiés à des dates différentes ${ }^{102}$, ce qui nous a permis de détecter des évolutions et d'établir des relations de filiation quand deux fiches-métiers comportent par exemple des missions similaires. Le seul métier que nous avons retrouvé dans tous les référentiels, que les fiches datent de 2009 ou de 2015 est celle du community manager (que nous nous permettrons d'abréger dans la suite par $\mathrm{CM}$ ). Nous supposons donc que c'est le plus établi des métiers du numérique possédant des compétences communicationnelles. C'est pourquoi nous l'avons choisi pour confronter plus précisément les fiches de chacun des référentiels.

En figure 4, nous proposons un tableau comparatif de l'ensemble de nos référentiels indiquant les missions que chaque fiche propose pour le $\mathrm{CM}$ : la fiche éditée en 2009 par Elaee, de laquelle nous avons extrait les missions, pour les comparer à celles citées dans trois autres référentiels, le portail des métiers de l'Internet, le référentiel des métiers de la communication et le référentiel de l'Apec publiés respectivement en 2012, 2013 et 2015.

\subsection{L'entretien des relations par du contenu conversationnel, une mission commune à tous les référentiels}

La première mission que nous avons retrouvée dans les fiches de tous les référentiels concerne la relation que l'animateur établit avec les membres de la communauté. L'animateur est un ambassadeur de la société qu'il représente. Il anime sa communauté en produisant des contenus et en incitant les membres à en produire aussi tout en modérant les conversations. Il possède donc une compétence rédactionnelle. Tous les référentiels considèrent que la rédaction de contenus fait partie des tâches incontournables sur les canaux numériques, mais certains référentiels, celui de l'Apec et celui de la communication insistent sur le fait que le $\mathrm{CM}$ incite les internautes à produire du contenu.

\footnotetext{
${ }^{102}$ Le référentiel de la communication a été publié en 2013. Le portail des métiers de 1'Internet comporte des dates de mises à jour pour certaines fiches. La plupart datent de 2012. Quand il n'y a pas de date de mise à jour, le commentaire le plus ancien donne une date approximative de la création de la fiche. Enfin le référentiel de l'Apec a été publié très récemment en juin 2015. Quant aux fiches métier d'Elaee, les premières ont été publiées dès 2009.
} 
Pour Elaee, le community manager « est l'évolution naturelle d'un poste de content manager» (Elaee, 2009), laissant supposer que ce métier de content manager (gestionnaire de contenus) préexiste au métier de community manager. A l'inverse, en 2015 l'Apec précise que

dans les entreprises qui ont atteint une maturité marketing digital, le poste en tant que tel n'existe souvent plus et les fonctions de community management sont alors éclatées entre d'autres postes (ex : social media manager, content manager) (Apec, 2015).

La multiplicité des missions du CM décrites dans la fiche Elaee et leur complexité croissante due à la diversité des médias sociaux entre 2009 et 2015 a favorisé l'émergence de nouveaux métiers qui sont des spécialisations ou des métiers plus stratégiques liés au community manager.

\subsection{Une mission d'e-réputation également attribuée à l'e- reputation manager et au gestionnaire des médias sociaux}

Elaee ajoute une mission d'e-réputation qui consiste à scruter « régulièrement les sites, forums, blogs en rapport avec l'activité de son entreprise », à en extraire les informations pertinentes et à proposer « un plan d'actions et des solutions en cas de problème (critique, crise, etc.) Pour ce faire, [le CM] instaure un dialogue avec les internautes pour résoudre le problème le plus rapidement possible et à moindre coût. » (Elaee, community manager, 2009).

Cette activité relève de la surveillance de l'e-réputation, qui consiste à surveiller ce que se dit sur l'annonceur sur les canaux numériques. Pour Alloing,

\footnotetext{
L'organisation pourra ainsi analyser son e-réputation comme une connaissance propre à une communauté donnée, et comme le reflet de la réalité que cette communauté à d'elle. Il n'est alors plus question d'opinions "négatives ou positives », mais d'un agencement d'opinions visant à construire ce qui régira par la suite les relations de cette communauté avec l'organisation. (Alloing, 2013, p. $312)$.
}

Cette mission apparaît aussi dans le portail des métiers de l'Internet pour le CM. Une surveillance régulière de ce qui se dit sur les réseaux est essentielle pour engager les conversations sur les sujets d'intérêt du moment.

Cette fonction est attribuée à d'autres métiers et dotée d'une dimension stratégique pour les référentiels de l'Apec et du portail des métiers de l'Internet. Elaee propose en plus de la fiche du CM une fiche pour le consultant e-réputation publié en 2012 dont le rôle 
consiste à parler et surtout faire parler de son entreprise, de son client ou de ses produits sur le web en intervenant à la fois sur la veille d'informations, la mise en place de contenus ainsi que la création et l'animation de communautés. Son objectif est clair : rendre l'opinion (informations, avis, échanges, commentaires, rumeurs, etc.) la plus favorable possible (Elaee, 2012).

Cette citation rend difficile la différenciation entre le $\mathrm{CM}$ et le consultant eréputation.

On peut néanmoins observer que le $\mathrm{CM}$ met en place une stratégie média social qui « repose sur une dualité : savoir s'adapter aux contenus des internautes et être capable d'enrichir la conversation » (Charest, Gauthier, \& Grenon, 2014, p. 273) en tenant compte de la spécificité du média et de la communauté choisie. Cette notion de stratégie pour le $\mathrm{CM}$ est limitée à la gestion des contenus sur un média, la prise en compte de la diversité des plateformes étant l'apanage du consultant e-réputation pour Elaee, du gestionnaire des médias sociaux pour le portail des métiers de l'Internet. Ce gestionnaire est chargé de mettre en place une stratégie de veille et d'actions sur les différents médias socionumériques en ligne.

Il intervient donc directement sur la e-réputation de l'organisation, sa visibilité auprès des différentes cibles (clients, partenaires, employés, candidats au recrutement....). Il a pour responsabilité de guider, conseiller la bonne utilisation des médias sociaux dans les différents champs de l'organisation : ressources humaines, communication, marketing, innovation, etc. (Portail des métiers de l'Internet, 2010)

Le référentiel de la communication associe quant à lui, l'e-reputation manager à un responsable de la communication externe on line.

Le consultant en e-réputation définit la politique de communication online en partenariat avec sa hiérarchie et les fonctions de relations publiques. Il élabore le plan de communication pour chaque projet et s'assure de la bonne réalisation des outils de communication définis. (Référentiel de la communication, 2013, Consultant e-reputation).

Ce consultant se préoccupe de la cohérence on-line off-line, les projets qu'il encadre pouvant être intégrés à des stratégies cross canal ${ }^{103}$.

\footnotetext{
${ }^{103}$ « le cross canal aborde l'ensemble des canaux de distribution et d'information de manière simultanée, afin de tirer profit des interactions et des synergies qui peuvent exister entre eux. L'approche cross canal permet ainsi de s'assurer de la cohérence de la promesse faite par la marque, qu'il convient alors de respecter au mieux d'un canal à l'autre afin de ne pas perturber l'acheteur potentiel. » disponible sur: http://www.e-marketing.fr/Definitions-Glossaire/Cross-canal-strategie-241173.htm
} 


\subsubsection{Des missions d'influence et d'efficacité}

Elaee attribue au CM une mission de visibilité sur les réseaux sociaux qu'il assure

par la création de groupes, de hubs ou de contenus viraux sur des sites tels que Facebook, Myspace, Viadeo, Youtube... (Elaee, community manager).

On ne retrouve pas nettement la mention de la viralité pour les fiches-métiers des autres référentiels, cette mission étant attribuée à d'autres fiches métiers. Le responsable des relations publiques digitales (Portail des métiers de l'Internet, 2013) analyse notamment le degré d'autorité attribué aux auteurs des jugements et les jeux d'influence en cours dans la communauté qu'il souhaite toucher. Les leaders d'opinion (les blogueurs, les journalistes, etc.) et les personnes qui relaient l'information vers des internautes influents sont des profils à détecter et à soigner car ils participent à la propagation du message. De plus, les jugements ou informations avancés par les internautes étant plus ou moins écoutés par leurs interlocuteurs, ils possèdent donc un degré d'influence plus ou moins fort. Camille Alloing définit un degré d'autorité qu'il désigne par l'expression «autorité informationnelle » qu'il assigne à des « agents facilitateurs » (Alloing \& PerdrieuMaudière, 2012). Les internautes prescripteurs d'informations sont des agents faciliteurs dans le sens où ils recontextualisent une information par la qualification de cette dernière et par le degré d'autorité, antérieur au message diffusé, qu'ils possèdent au sein d'une communauté.

De fait, l'annonceur doit, en plus de surveiller ce qui se dit, analyser le degré d'autorité attribuée aux auteurs des jugements et les jeux d'influence en cours dans la communauté qu'il souhaite toucher.

La mission dévolue au responsable des relations publiques digitales est donc stratégique et elle est complémentaire au travail du community manager. Ce responsable est un expert des relations publiques.

Il reste une mission que nous avons déjà bien présentée dans la partie précédente, celle de la mesure de l'efficacité des actions. Elle n'est pas mentionnée dans la fiche Elaee, ni dans celle du portail des métiers de l'Internet qui concerne le CM, ce dernier la décrivant clairement en revanche pour le chef de projet e-crm.

Le chef de projet e-CRM a trois missions majeures : l'amélioration de la connaissance du client, la fidélisation du client en ligne et le développement de la valeur du client. Il définit la stratégie d'exploitation des données des visiteurs et/ou clients. Il construit et assure la mise en œuvre des solutions e-CRM pour proposer des produits adaptés au profil du visiteur. Il mesure la performance et le retour sur investissement des actions menées.(Portail des métiers de l'internet, Chef de projet e-crm, >oct 2013) 
148 Le communicateur bousculé par le numérique

Cette fiche comporte une dimension relationnelle instaurée entre le client et l'annonceur mais celle-ci est clairement à connotation marketing puisque les données récupérées par les échanges conversationnels ou marchands sont utiles pour réorienter les actions marketing.

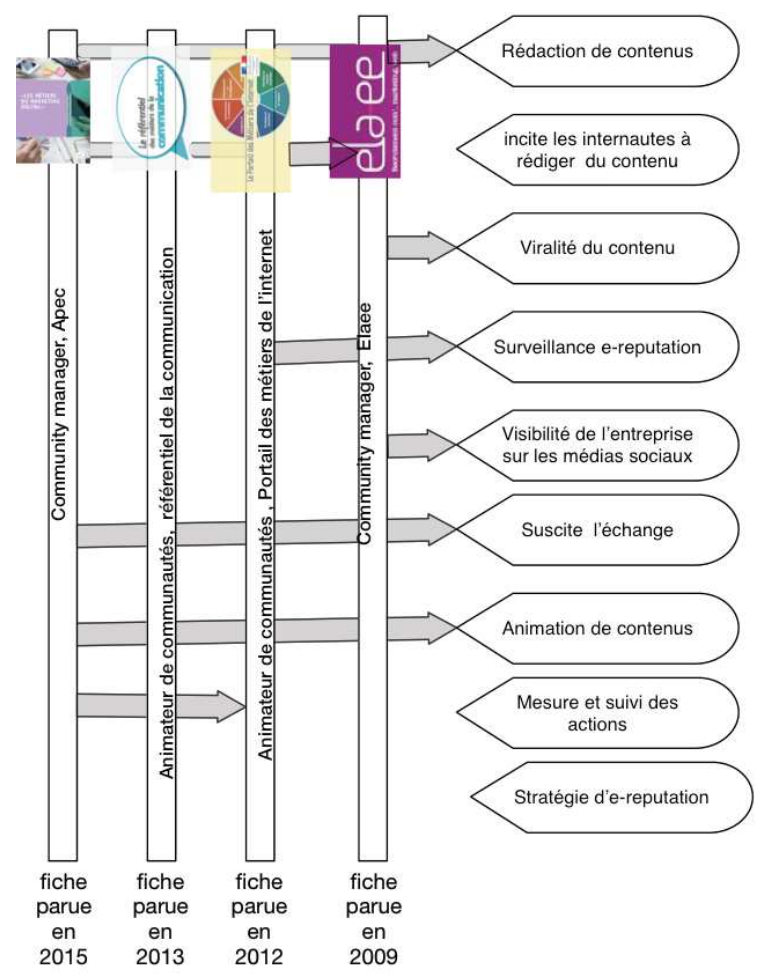

\section{FIGURE 15}

\section{Confrontation des missions du community manager dans quatre référentiels}

Finalement, l'ensemble des missions assignées au community manager par Elaee, assorti de la mission de mesure des actions, le rapproche du social media manager (responsable des medias ou réseaux sociaux), décrit par l'Apec. Il a

pour mission de développer une stratégie de présence numérique de l'entreprise sur les différents médias sociaux pour les clients et prospects. Il accompagne sa mise en oeuvre afin d'assurer une bonne visibilité, générer du trafic et surveiller l'e-réputation de l'entreprise (référentiel Apec, 2015). 
Nous retrouvons les missions de visibilité et d'e-reputation. Nous n'avons pas mentionné jusqu'ici la préoccupation concernant le trafic, pourtant présente dans la fiche Elaee.

Il peut intervenir sur le développement de la notoriété de sa société par la réalisation d'actions webmarketing : référencement du site, netlinking, partenariats entre sites web, affiliation... (Elaee, community manager, 2009)

Elaee possède une autre fiche pour détailler les missions du traffic manager qui consiste à :

Optimiser les leviers d'acquisition de trafic sur un (ou plusieurs) site(s) web afin d'attirer un trafic qualifié et de maximiser les ventes ou de recruter de nouveaux prospects. Référencement payant, référencement naturel, e-mailing, affiliation, display, comparateurs de prix... ${ }^{104} \mathrm{Il}$ consiste aussi «à mesurer avec précision l'audience de son site en utilisant différents indicateurs: nombre de visiteurs uniques, pages vues, parcours et durée des visites, nombre de clics, mots saisis dans le moteur de recherche interne du site... (Elaee, traffic manager, 2009)

Ce métier est cité par l'ensemble des référentiels, qui lui assigne les mêmes missions, même si les technologies évoluent. Le traffic manager travaille en étroite collaboration avec les social media manager.

La dimension marketing du CM apparait nettement dans le référentiel de la communication et dans celui de l'Apec. Elle est clairement attribuée au social media manager qui gère l'aspect marketing par la mise en place de dispositifs sociaux impactants du type jeux concours, applications et de dispositifs de mesure qui permettent de mesurer l'efficacité des actions.

Le social media manager possède une vision plus gestionnaire et clairement orientée sur les stratégies de performance et d'efficacité marketing. C'est lui qui impose au CM des contraintes marketing.

Finalement, la confrontation des fiches-métier du community manager de différents référentiels permet d'aborder d'autres métiers qui sont des spécialisations. Si en 2009 Elaee assigne au CM l'ensemble des fonctions que nous avons discutées dans les fiches-métiers de 2013, des métiers spécialisés apparaissent pour prendre en compte les différentes communautés (intérêt pour une communauté particulière réduite à une cible - les influenceurs pour le responsable des relations publiques digitales, les clients pour le chef de projet e-crm) et la diversité des médias socionumériques. Enfin en 2015, c'est surtout l'apparition du mobile qui est une nouveauté.

\footnotetext{
${ }^{104}$ Cette fiche ne comportant pas de date de publication, ni de commentaire, nous avons indiqué une date supérieure à 2009, date à laquelle les fiches-métiers ont démarré sur le blog d'Elaee.
} 
On peut constater à l'issue de cette analyse que l'ensemble des compétences attendues sont finalement plus stables que la pléthore de noms de métiers naissant chaque année. La viralité, l'e-réputation, la cohérence entre le on-line et le off-line, la stratégie de présence sur les médias socionumériques, les stratégies d'influence et de visibilité, la génération de trafic, sont les missions essentielles qui traversent l'ensemble des métiers étudiés.

Notre analyse soulève aussi l'obsession très gestionnaire de discrétiser toutes les compétences en sous-métiers, leur faisant perdre une vision globale permettant de construire une véritable stratégie de communication digitale et permettant ainsi de concevoir une véritable profession. En 2009, les métiers avaient une coloration plutôt opérationnelle. Des métiers stratégiques se sont développés depuis, montrant notamment la prise en compte d'une vision multicanaux depuis 2013 (voir le responsable de la communication on-line) ou l'intégration de différents médias socionumériques (gestionnaire de médias sociaux) aux stratégies de communication. Mais la stratégie globale est plutôt laissée au responsable marketing exigeant des communicateurs des priorités d'efficacité immédiate, incompatibles avec la construction d'une relation durable et authentique.

\title{
4. Conclusion
}

Notre étude a mis en exergue la richesse et la diversité des métiers en émergence dans le secteur du numérique. Elle a permis d'étudier la dynamique des métiers liés à la communication, en se limitant toutefois à la vision des auteurs de référentiels.

Cette étude exclut la vision des professionnels au contact quotidien avec les internautes qui développent des habiletés professionnelles (Larroche, Piment, 2014). Ajouter la représentation des professionnels experts du numérique aux référentiels et celle des responsables des ressources humaines des services de formation de salariés et de recrutement permettrait d'évaluer le degré de professionnalisation de ces métiers. La professionnalisation renvoie

\begin{abstract}
Au sens anglo-saxon (Bourdoncle, 1991), à une dynamique d'autonomisation, de reconnaissance sociale et statutaire par un groupe socio-professionnel donné qui s'organise et cherche à se protéger par une formation spécifique, un ordre professionnel propre, la caractérisation de compétences spécifiques et d'un champ d'activités qui lui soit réservé et dont l'accès est contrôlé par la détention d'un diplôme particulier. (Maubant, Piot, 2011, p. 8).
\end{abstract}

Le métier, nous l'avons dit, n'est pas réfléchi ici en terme d'identité collective (Osty, 2003) mais confronte des discours qui permettent à des demandeurs d'emploi 
ou des employeurs d'identifier des dénominations de poste ${ }^{105}$ et les compétences correspondantes. C'est un outil de traduction (Callon, 1986) au même titre que les maquettes pédagogiques (Larroche, 2014), et les réseaux sociaux professionnels numériques (Larroche, 2015) qui participent à la définition des stratégies d'acteurs.

Notre analyse a permis de constater la place des compétences infocommunicationnelles dans les métiers émergents du numérique, compétences limitées malgré tout à une vision très marketing de la communication due au fait que les référentiels étudiés valorisent la représentation des employeurs des grandes entreprises et sont inspirés des instruments de gestion des compétences associés aux techniques managériales au service de la rationalisation (Dietrich, 1999).

La relation authentique et l'interaction sont souvent négligées quand le primat est donné à la communication de marque et à la seule visibilité. Un des points ignorés dans cet article, et pourtant indispensable, est la diversité des positionnements de l'entreprise. Tantôt annonceur - le seul point de vue de l'organisation présenté dans cet article, agent institutionnel, acteur-citoyen (responsabilité sociale de l'entreprise), responsable d'une communauté de salarié, etc., ces positionnements impliquent des missions pour le communicateur qui, pour certains permettent le développement d'une « communication de proximité, nécessaire à la confiance et à la reconnaissance mutuelle» (De la Broise, Morillon, 2014, p. 170).

\section{Bibliographie}

Alloing, C., \& Perdrieu-Maudière, F. (2012). De surveiller à «prendre soin»: comment repenser la veille sur les réseaux sociaux numériques en termes de management de réseaux d'acteurs? Revue internationale d'intelligence économique, 4(1), 55-70. doi:10.3166/r2ie.4.55-70

Berland N., Chevalier-Kuszla C., Sponem S. (2008), On ne gère bien que ce que l'on mesure, In Petit bréviaire des idées reçues en management, Paris, La Découverte.

\footnotetext{
${ }^{105}$ Voir à cet égard le document de l'Apec, «Les métiers en émergence au travers des offres d'emploi, exploitation des offres d'emploi publiées par l'Apec sur les métiers dits « en émergence » entre 2010 et le 1er semestre 2014 », Les études de l’emploi cadre Apec, n 2015-2, janvier 2015, p.8, disponible sur: http://www.cticommission.fr/IMG/pdf/les_metiers_en_emergence_au_travers_des_offres_apec.pdf. Dans ce document, les métiers de chargé de communication on line, de community manager et de consultant en e-réputation sont considérés comme des métiers en émergence au vu du volume d'offres d'emplois publiées.
} 
Bouquet, B. (2014), La complexité de la légitimité, Vie sociale, Vol. 4, n 8, p. 1323.

Bourdoncle, R. (1991). La professionnalisation des enseignants: analyses sociologiques anglaises et américaines. Consulté à l'adresse http://www.iamm.fr/ress_doc/opac_css/index.php?lvl=notice_display\&id=2585

Brulois, V., \& Charpentier, J.-M. (2013). Refonder la communication en entreprise, Limoges: FYP éditions.

Callon M. (1986), Éléments pour une sociologie de la traduction. La domestication des coquilles Saint-Jacques et des marins-pêcheurs dans la baie de Saint-Brieuc, L’Année Sociologique, n³6, p. 169-208.

Carmes, M., \& Galibert, O. (2012). Discriminations numériques en organisation: une analyse des identités connectives et des revendications au sein d'une grande entreprise française. In B. Cordelier \& G. Gramaccia (Éd.), Management par projet: Les identités incertaines, Québec, Presses de 1'Université du Québec, p. 81-100.

Charest, F., Gauthier, A.-M., \& Grenon, F. (2014), Appropriation et stratégies d'intégration des médias sociaux par les professionnels de la communication, Communication \& Organisation, Vol. 1, n 43, p. 269-280.

Dacheux É., Étudier le marketing à la lumière de la communication, L'Année sociologique, 2001, n², Vol. 51, p. 411-427.

Debos, F. (2008). Les relations numériques individu-marque. Document numérique, Vol.3, n¹0, p. 63-73.

De la Broise, P. (2013). La lutte pour la reconnaissance: Codes, chartes, référentiels et autres manifestes des professionnels français de la communication, Reciproc, $\mathrm{n}^{\circ}$ 1: La professionnalisation des communicateurs: Dynamiques, tensions et vecteur, p. 33-50.

De la Broise, P. (2014), Des pratiques aux formations professionnalles, les liaisons dangereuses du marketing et de la communication, in V. Lépine, F. MartinJuchat, C. Millet-Fourrier, Acteurs de la communication des entreprises et organisations, pratiques et perspectives, Grenoble, PUG, p. 165-179.

Dietrich A. (1999), La dynamique des compétences, point aveugle des techniques managériales, Formation emploi, ${ }^{\circ}$ 67, p. 9-23.

Galibert, O. (2014). Vers une instrumentalisation généralisée du lien communautaire en ligne: la montée en puissance du community management. In Acteurs de la communication des entreprises et organisations, pratiques et perspectives, Grenoble, PUG, p. 117-136. 
Girard, B. (2012). Le souci de sa réputation peut - il modifier les comportements d'une entreprise? disponible sur http://www.dissonancesx90x.net/Reputation.pdf

Granget L. (2012), Le communicant d'université : un modèle professionnel (dé)bridé, Communication \& Organisation, vol.1, n 41, p. 181-194.

Grunig, J. E., \& Hunt, T. (1984), Managing public relations, Fort Worth, Etats-Unis.

Heinich, N. (2012). De la visibilité: excellence et singularité en régime médiatique, Paris, Gallimard.

Hossler, M., Murat, O., \& Jouanne, A. (2014). Faire du marketing sur les réseaux sociaux, Paris, Eyrolles.

Karpik, L. (2007). L'économie des singularités, Paris, Gallimard.

Kittel I. (2014), Les référentiels, supports ou obstacles à la professionnalisation ?, Empan, Vol. 3, n 95, p. 42-48.

Larroche V. (2015), Les médiateurs de confiance comme gage de crédibilité des candidats : Le cas des réseaux sociaux professionnels numériques, In $\mathrm{C}$. Alcantara, E-reputation : regards croisés sur une notion émergente, Gualino Editions, p.145-156.

Larroche V. (2014), Innovation organisationnelle dans la conception d'un diplôme universitaire professionnel dans le secteur de la communication digitale, Reciproc, $n^{\circ}$ 2, UCL Presses universitaire de Louvain, p. 53-74.

Larroche V., Piment H. (2014) De l'habileté dans les interactions numériques aux compétences professionnelles de l'animateur de communautés en ligne, In : 1112-13 juin 2014. Colloque Travail et loisirs ou comment le travail se transforme par et dans le divertissement et comment le divertissement se requalifie en travail? Neuilly sur Seine : GRIPIC, Celsa Paris-Sorbonne, p. 159-168

Lépine V., Peyrelong M.-F. (2012), Aux frontières de deux métiers : les compétences communicationnelles en jeu dans les bibliothèques, Communication et organisation [En ligne], $\mathrm{n}^{\circ} 41$, mis en ligne le 01 juin 2015, URL : http://communicationorganisation.revues.org/3730

Maubant, P., \& Piot, T. (2011). Étude des processus de professionnalisation dans les métiers adressés à autrui, Les Sciences de l'éducation, Pour l'Ère nouvelle, $\mathrm{n}^{\circ} 44$, vol 2, p. 7.

Origgi, G. (2013). Un certain regard. Communications, $\mathrm{n}^{\circ}$ 93(2), 101-120. doi:10.3917/commu.093.0101 
154 Le communicateur bousculé par le numérique

Osty, F. (2003). Le désir de métier: engagement, identité et reconnaissance au travail, Rennes, Presses universitaires de Rennes.

Stenger T., Coutant A. (2011), Community management et community managers : Cheval de Troie marketing pour le web social? In Web social, communautés virtuelles et consommation. 2011

Stenger, T., Coutant, A. (2013). Médias sociaux: clarification et cartographie - Pour une approche sociotechnique. Décisions Marketing, $\mathrm{n}^{\circ}$ 70, 107-117.

Trépos J-Y. (1992), Sociologie de la compétence professionnelle, Nancy, Presse Universitaire de Nancy.

Voirol O. (2005), Les luttes pour la visibilité. Esquisse d'une problématique, Réseaux, Vol.1, n¹29-130, p. 89-121. 


\section{Annexe : Les références des annuaires constitutifs du corpus}

(Référentiel de la communication, 2013), AACC, AFCI, ANAE, Club des annonceurs, Communication \& Entreprise, Communication publique, IP\&C, SYNAP, Syntec Conseil en Relations Publics, UDA, Le référentiel des métiers de la communciation, 2013, [en ligne], disponible sur : http://metiersdelacommunication.fr/ (consulté le 29 juillet 2015).

(Apec, 2015), Apec, Référentiel des métiers du marketing digital, juin 2015, disponible sur: https://cadres.apec.fr/Emploi/Marche-Emploi/Les-EtudesApec/Les-etudes-Apec-par-annee/Etudes-Apec-2015/Referentiel-des-metiersdu-marketing-digital/Marketing-digital-des-metiers-cadres-en-constantemutation

(Apec, 2013), Apec, Référentiel des métiers en émergence, hors-série, avril 2013 disponible

sur : https://cadres.apec.fr/Emploi/content/download/495317/1057329/version/1/file/ Metiers_en_emergence.pdf

(Portail des métiers de l'Internet), Délégation aux Usages de l'Internet, Le portail des métiers de l'Internet, [en ligne], disponible sur : http://metiers.internet.gouv.fr/(consulté le 29 juillet 2015).

(Elaee), Elaee, fiches-métiers, [en ligne], disponible sur : http://www.elaee.com/fiches-metiers (consulté le 29 juillet 2015)

(IAB France, 2015), IAB France (Interactive Advertising Bureau France), Etude sur les métiers et les compétences : de la transition numérique dans le secteur du marketing et de la communication, Février 2015, disponible sur : http://www.iabfrance.com/presse/communiques-de-presse/les-metiers-dumarketing-et-de-la-communication 